\title{
ON THE PRODUCT AND RATIO OF LAPLACE AND BESSEL RANDOM VARIABLES
}

\author{
SARALEES NADARAJAH
}

Received 4 November 2004 and in revised form 8 February 2005

The distributions of products and ratios of random variables are of interest in many areas of the sciences. In this paper, the exact distributions of the product $|X Y|$ and the ratio $|X / Y|$ are derived when $X$ and $Y$ are Laplace and Bessel function random variables distributed independently of each other.

\section{Introduction}

For given random variables $X$ and $Y$, the distributions of the product $|X Y|$ and the ratio $|X / Y|$ are of interest in many areas of the sciences.

In traditional portfolio selection models, certain cases involve the product of random variables. The best examples of this are in the case of investment in a number of different overseas markets. In portfolio diversification models (see, e.g., Grubel [7]), not only are prices of shares in local markets uncertain but also the exchange rates are uncertain so that the value of the portfolio in domestic currency is related to a product of random variables. Similarly in models of diversified production by multinationals (see, e.g., Rugman [23]), there is local production uncertainty and exchange rate uncertainty so that profits in home currency are again related to a product of random variables. An entirely different example is drawn from the econometric literature. In making a forecast from an estimated equation, Feldstein [5] pointed out that both the parameter and the value of the exogenous variable in the forecast period could be considered as random variables. Hence, the forecast was proportional to a product of random variables.

An important example of ratios of random variables is the stress-strength model in the context of reliability. It describes the life of a component which has a random strength $Y$ and is subjected to random stress $X$. The component fails at the instant that the stress applied to it exceeds the strength and the component will function satisfactorily whenever $Y>X$. Thus, $\operatorname{Pr}(X<Y)$ is a measure of component reliability. It has many applications especially in engineering concepts such as structures, deterioration of rocket motors, static fatigue of ceramic components, fatigue failure of aircraft structures, and the aging of concrete pressure vessels. 
The distributions of $|X Y|$ and $|X / Y|$ have been studied by several authors especially when $X$ and $Y$ are independent random variables and come from the same family. With respect to products of random variables, see Sakamoto [24] for uniform family, Harter [8] and Wallgren [30] for Student's $t$ family, Springer and Thompson [26] for normal family, Stuart [28] and Podolski [16] for gamma family, Steece [27], Bhargava and Khatri [3], and Tang and Gupta [29] for beta family, Abu-Salih [1] for power function family, and Malik and Trudel [13] for exponential family (see also Rathie and Rohrer [22] for a comprehensive review of known results). With respect to ratios of random variables, see Marsaglia [14] and Korhonen and Narula [10] for normal family, Press [17] for Student's $t$ family, Basu and Lochner [2] for Weibull family, Shcolnick [25] for stable family, Hawkins and Han [9] for noncentral chi-squared family, Provost [18] for gamma family, and Pham-Gia [15] for beta family. There is relatively little work of the above kind when $X$ and $Y$ belong to different families. In the applications mentioned above, it is quite possible that $X$ and $Y$ could arise from different but similar distributions (see below, e.g.).

In this paper, we derive the exact distributions of $|X Y|$ and $|X / Y|$ when $X$ and $Y$ are independent random variables having the Laplace and Bessel function distributions with pdfs

$$
\begin{gathered}
f(x)=\frac{\lambda}{2} \exp (-\lambda|x|), \\
f(y)=\frac{\left|1-c^{2}\right|^{m+1 / 2}|y|^{m}}{\sqrt{\pi} 2^{m} b^{m+1} \Gamma(m+1 / 2)} \exp \left(-\frac{c y}{b}\right) K_{m}\left(\left|\frac{y}{b}\right|\right),
\end{gathered}
$$

respectively, for $-\infty<x<\infty,-\infty<y<\infty, \lambda>0, b>0,|c|<1$, and $m>1$, where

$$
K_{m}(x)=\frac{\sqrt{\pi} x^{m}}{2^{m} \Gamma(m+1 / 2)} \int_{1}^{\infty}\left(t^{2}-1\right)^{m-1 / 2} \exp (-x t) d t
$$

is the modified Bessel function of the third kind. Several of the more standard distributions in statistics are particular cases of (1.2) for integer and half-integer values of $m$. Thus, we also provide particular cases of our results for integer and half-integer values of $m$.

Laplace and Bessel function distributions have found applications in a variety of areas that range from image and speech recognition and ocean engineering to finance. Both are rapidly becoming distributions of first choice whenever "something" with heavier than Gaussian tails is observed in the data. Some examples are the following (see Kotz et al. [11] for further applications):

(1) in communication theory, $X$ and $Y$ could represent the random noise corresponding to two different signals,

(2) in ocean engineering, $X$ and $Y$ could represent distributions of navigation errors,

(3) in finance, $X$ and $Y$ could represent distributions of logreturns of two different commodities,

(4) in image and speech recognition, $X$ and $Y$ could represent "input" distributions.

In each of the examples above, it will be of interest to study the distribution of the ratio $|X / Y|$. For example, in communication theory, $|X / Y|$ could represent the relative 
strength of the two different signals. In ocean engineering, $|X / Y|$ could represent the relative safety of navigation. In finance, $|X / Y|$ could represent the relative popularity of the two different commodities. The distribution of the product $|X Y|$ is considered here for completeness.

The exact expressions for the distributions of the product and ratio are given in Sections 2 and 3 of the paper. The calculations involve the Bessel function of the first kind defined by

$$
J_{\nu}(x)=\frac{x^{\nu}}{2^{\nu} \Gamma(\nu+1)} \sum_{k=0}^{\infty} \frac{1}{(\nu+1)_{k} k !}\left(-\frac{x^{2}}{4}\right)^{k},
$$

the modified Bessel function of the first kind defined by

$$
I_{\nu}(x)=\frac{x^{\nu}}{2^{\nu} \Gamma(\nu+1)} \sum_{k=0}^{\infty} \frac{1}{(\nu+1)_{k} k !}\left(\frac{x^{2}}{4}\right)^{k},
$$

and the hypergeometric functions defined by

$$
\begin{aligned}
& { }_{0} F_{3}(a, b, c ; x)=\sum_{k=0}^{\infty} \frac{1}{(a)_{k}(b)_{k}(c)_{k}} \frac{x^{k}}{k !}, \\
& { }_{2} F_{1}(a, b ; c ; x)=\sum_{k=0}^{\infty} \frac{(a)_{k}(b)_{k}}{(c)_{k}} \frac{x^{k}}{k !},
\end{aligned}
$$

where $(e)_{k}=e(e+1) \cdots(e+k-1)$ denotes the ascending factorial. The properties of the above special functions can be found in Lebedev [12], Erdelyi et al. [4], Prudnikov et al. $[19,20,21]$, and Gradshteyn and Ryzhik [6].

\section{Product}

Theorem 2.1 derives an explicit expression for the cdf of $|X Y|$ in terms of the hypergeometric functions.

Theorem 2.1. Suppose $X$ and $Y$ are distributed according to (1.1) and (1.2), respectively, with $c=0$. The cdf of $Z=|X Y|$ can be expressed as

$$
\begin{aligned}
F(z)=1- & \left\{\sqrt{\pi} 2^{m} b^{m+1} \Gamma\left(m+\frac{1}{2}\right){ }_{0} F_{3}\left(\frac{1}{2}, \frac{1}{2}-m, \frac{1}{2} ; \frac{\lambda^{2} z^{2}}{16 b^{2}}\right)\right. \\
& +(2 b)^{-m}(\lambda z)^{2 m+1} \Gamma(-m) \Gamma(-2 m-1)_{0} F_{3}\left(1+m, \frac{3}{2}+m, 1+m ; \frac{\lambda^{2} z^{2}}{16 b^{2}}\right) \\
& \left.\quad+\left(\frac{3 C}{2}-1\right)(2 b)^{m} \lambda z \Gamma(m)_{0} F_{3}\left(1-m, \frac{3}{2}, 1 ; \frac{\lambda^{2} z^{2}}{16 b^{2}}\right)\right\} \\
& \times \frac{1}{\sqrt{\pi} 2^{m} b^{m+1} \Gamma(m+1 / 2)},
\end{aligned}
$$

where $C$ denotes the Euler constant. 
396 On the product and ratio of Laplace and Bessel random variables

Proof. The $\operatorname{cdf} F(z)=\operatorname{Pr}(|X Y| \leq z)$ can be expressed as

$$
\begin{aligned}
F(z) & =\operatorname{Pr}(|X| \leq z /|Y|) \\
& =\frac{1}{\sqrt{2} 2^{m} b^{m+1} \Gamma(m+1 / 2)} \int_{-\infty}^{\infty}\left\{1-\exp \left(-\frac{\lambda z}{|y|}\right)\right\}|y|^{m} K_{m}\left(\left|\frac{y}{b}\right|\right) d y \\
& =1-\frac{1}{\sqrt{2} 2^{m} b^{m+1} \Gamma(m+1 / 2)} \int_{-\infty}^{\infty} \exp \left(-\frac{\lambda z}{|y|}\right)|y|^{m} K_{m}\left(\left|\frac{y}{b}\right|\right) d y \\
& =1-\frac{1}{\sqrt{2} 2^{m-1} b^{m+1} \Gamma(m+1 / 2)} \int_{0}^{\infty} \exp \left(-\frac{\lambda z}{y}\right) y^{m} K_{m}\left(\frac{y}{b}\right) d y
\end{aligned}
$$

The result of the theorem follows by applying the integration formula (Prudnikov et al. [20, equation $(2.16 .8 .9)])$ that

$$
\begin{aligned}
& \int_{0}^{\infty} x^{\alpha-1} \exp \left(-\frac{p}{x}\right) K_{v}(c x) d x \\
& =\frac{2^{\alpha-2}}{c^{\alpha}} \Gamma\left(\frac{\alpha+v}{2}\right) \Gamma\left(\frac{\alpha-v}{2}\right){ }_{0} F_{3}\left(\frac{1}{2}, 1-\frac{\alpha+v}{2}, 1-\frac{\alpha-v}{2} ; \frac{c^{2} p^{2}}{16}\right) \\
& -\frac{2^{\alpha-3} p}{c^{\alpha-1}} \Gamma\left(\frac{\alpha+v-1}{2}\right) \Gamma\left(\frac{\alpha-v-1}{2}\right){ }_{0} F_{3}\left(\frac{3}{2}, \frac{3-\alpha-v}{2}, \frac{3+v-\alpha}{2} ; \frac{c^{2} p^{2}}{16}\right) \\
& +\frac{p^{\alpha+v} c^{v}}{2^{v+1}} \Gamma(-v) \Gamma(-v-\alpha)_{0} F_{3}\left(1+v, 1+\frac{\alpha+v}{2}, \frac{1+\nu+\alpha}{2} ; \frac{c^{2} p^{2}}{16}\right) \\
& +\frac{p^{\alpha-\nu}}{2^{1-\nu} c^{\nu}} \Gamma(\nu) \Gamma(\nu-\alpha)_{0} F_{3}\left(1-\nu, 1+\frac{\alpha-\nu}{2}, \frac{1+\alpha-\nu}{2} ; \frac{c^{2} p^{2}}{16}\right)
\end{aligned}
$$

(for $c>0$ and $p>0$ ) to calculate the integral in (2.2).

Using the special property of the ${ }_{0} F_{3}$ hypergeometric function (Prudnikov et al. [21, equation (7.16.2.9)]) that

$$
\begin{aligned}
& { }_{0} F_{3}\left(n, n-m-\frac{1}{2}, n-m-l-\frac{1}{2} ; z\right) \\
& \quad=\frac{(-1)^{l}(n-1) !\left((1 / 2)_{n-1}\right)^{2} z^{l+m-n+3 / 2}}{2\left((3 / 2-n)_{m}\right)^{2}(3 / 2+m-n)_{l}}\left(\frac{d}{d z}\right)^{l}\left(\frac{d}{d z} z \frac{d}{d z}\right)^{m} z^{n-3 / 2}\left(\frac{d}{d z}\right)^{n-1}\left\{J_{0}(x)+I_{0}(x)\right\},
\end{aligned}
$$




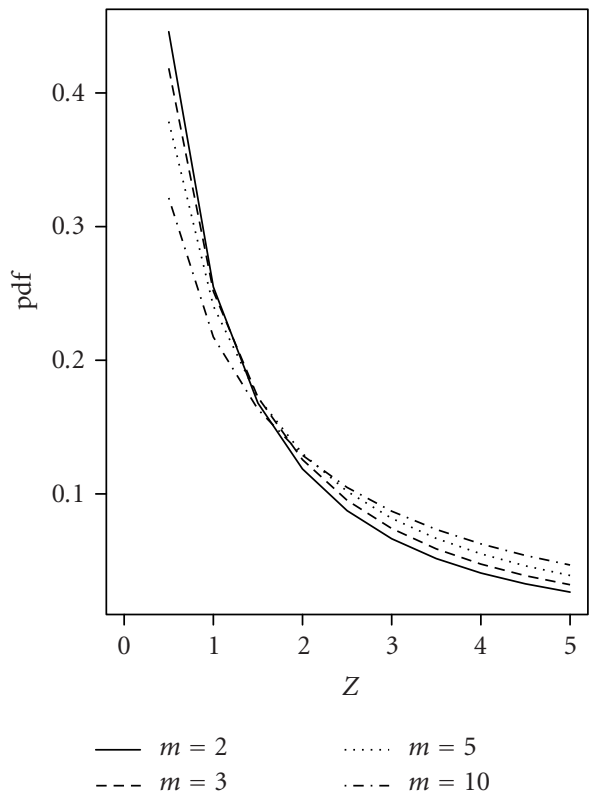

Figure 2.1. Plots of the pdf of (2.1) for $\lambda=1, b=1$, and $m=2,3,5,10$.

one can derive simpler forms for the distribution of $|X Y|$ when $m$ takes half integer values. For example, if $m=3 / 2$ and $m=5 / 2$, then (2.1) can be reduced to

$$
\begin{aligned}
F(z)=-\frac{1}{8 y}\{ & -8 y-4 I_{0}(0,2 y) y-3 I_{0}(2 y) y^{3} C+2 I_{0}(2 y) y^{3}-4 J_{0}(2 y) y \\
& -3 J_{0}(y) y^{3} C+2 J_{0}(2 y) y^{3}+8 J_{1}(2 y)+6 I_{1}(2 y) y^{2} C-4 I_{1}(2 y) y^{2} \\
& \left.+8 J_{1}(2 y)+6 J_{1}(2 y) y^{2} C-4 J_{1}(2 y) y^{2}\right\}, \\
F(z)=-\frac{1}{96 y}\{ & -96 y-80 I_{0}(2 y) y-45 I_{0}(2 y) y^{3} C+30 I_{0}(2 y) y^{3}-80 J_{0}(2 y) y \\
& -45 J_{0}(2 y) y^{3} C+30 J_{0}(2 y) y^{3}+128 I_{1}(2 y)+72 I_{1}(2 y) y^{2} C \\
& -32 I_{1}(2 y) y^{2}+9 I_{1}(2 y) y^{4} C-6 I_{1}(2 y) y^{4}+128 J_{1}(2 y) \\
& \left.+72 J_{1}(2 y) y^{2} C-64 J_{1}(2 y) y^{2}-9 J_{1}(2 y) y^{4} C+6 J_{1}(2 y) y^{4}\right\},
\end{aligned}
$$

respectively, where $y=\sqrt{\lambda z / b}$ and $C$ denotes the Euler constant.

Figure 2.1 illustrates possible shapes of the pdf of (2.1) for $\lambda=1, b=1$, and a range of values of $m$. Note that the shapes are unimodal and that the value of $m$ largely dictates the behavior of the pdf near $z=0$.

\section{Ratio}

Theorem 3.1 derives an explicit expression for the cdf of $|X / Y|$ in terms of the hypergeometric functions. 
398 On the product and ratio of Laplace and Bessel random variables

Theorem 3.1. Suppose $X$ and $Y$ are distributed according to (1.1) and (1.2), respectively. The cdf of $Z=|X / Y|$ can be expressed as

$$
\begin{aligned}
& F(z)=1-\frac{\left|1-c^{2}\right|^{m+1 / 2} \Gamma(2 m+1)}{\sqrt{\pi} 2^{2 m+1} \Gamma(m+1 / 2) \Gamma(m+3 / 2)} \\
& \times\left\{\frac{1}{\lambda b z-c}{ }_{2} F_{1}\left(\frac{1}{2}, 1 ; m+\frac{3}{2} ; 1-\frac{1}{(\lambda b z-c)^{2}}\right)\right. \\
&\left.+\frac{1}{\lambda b z+c}{ }_{2} F_{1}\left(\frac{1}{2}, 1 ; m+\frac{3}{2} ; 1-\frac{1}{(\lambda b z+c)^{2}}\right)\right\} .
\end{aligned}
$$

Proof. The $\operatorname{cdf} F(z)=\operatorname{Pr}(|X / Y| \leq z)$ can be expressed as

$$
\begin{aligned}
F(z)= & \frac{\left|1-c^{2}\right|^{m+1 / 2}}{\sqrt{\pi} 2^{m} b^{m+1} \Gamma(m+1 / 2)} \\
& \times \int_{-\infty}^{\infty}\{F(z|y|)-F(-z|y|)\}|y|^{m} \exp \left(-\frac{c y}{b}\right) K_{m}\left(\left|\frac{y}{b}\right|\right) d y,
\end{aligned}
$$

where $F(\cdot)$ inside the integral denotes the cdf corresponding to $(1.1)$ given by

$$
F(x)= \begin{cases}\frac{1}{2} \exp (\lambda x), & \text { if } x \leq 0 \\ 1-\frac{1}{2} \exp (-\lambda x), & \text { if } x>0\end{cases}
$$

Substituting (3.3) for $F(\cdot)$, one can rewrite (3.2) as

$$
\begin{aligned}
F(z)= & \frac{\left|1-c^{2}\right|^{m+1 / 2}}{\sqrt{\pi} 2^{m} b^{m+1} \Gamma(m+1 / 2)} \\
& \times\left[\int_{-\infty}^{0}\{1-\exp (\lambda z y)\}|y|^{m} \exp \left(-\frac{c y}{b}\right) K_{m}\left(\left|\frac{y}{b}\right|\right) d y\right. \\
& \left.\quad+\int_{0}^{\infty}\{1-\exp (-\lambda z y)\}|y|^{m} \exp \left(-\frac{c y}{b}\right) K_{m}\left(\left|\frac{y}{b}\right|\right) d y\right] \\
= & 1-\frac{\left|1-c^{2}\right|^{m+1 / 2}}{\sqrt{\pi} 2^{m} b^{m+1} \Gamma(m+1 / 2)} \\
& \times\left[\int_{0}^{\infty} y^{m} \exp \left(-\lambda z y+\frac{c y}{b}\right) K_{m}\left(\frac{y}{b}\right) d y+\int_{0}^{\infty} y^{m} \exp \left(-\lambda z y-\frac{c y}{b}\right) K_{m}\left(\frac{y}{b}\right) d y\right] .
\end{aligned}
$$


The result in (3.1) follows by applying the integration formula (Prudnikov et al. [20, equation $(2.16 .6 .3)]$ ) that

$$
\begin{aligned}
& \int_{0}^{\infty} x^{\alpha-1} \exp (-p x) K_{\nu}(c x) d x \\
& \quad=\frac{p^{\nu-\alpha} \sqrt{\pi} \Gamma(\alpha-\nu) \Gamma(\alpha+\nu)}{2^{\alpha} c^{\nu} \Gamma(\alpha+1 / 2)}{ }_{2} F_{1}\left(\frac{\alpha-\nu}{2}, \frac{\alpha-\nu+1}{2} ; \alpha+\frac{1}{2} ; 1-\frac{c^{2}}{p^{2}}\right)
\end{aligned}
$$

(for $c+p>0$ and $\alpha>v$ ) to calculate the two integrals in (3.4).

Using special properties of the ${ }_{2} F_{1}$ hypergeometric function (Prudnikov et al. [21, equations (7.3.1.137) and (7.3.1.124)]) that

$$
\begin{gathered}
{ }_{2} F_{1}\left(1, \frac{1}{2} ; m+\frac{1}{2} ; z\right)=\frac{(1 / 2)_{m}(z-1)^{m-1}}{(m-1) ! z^{m}}\left[2 \sqrt{z} \operatorname{arctanh}(\sqrt{z})+\sum_{k=1}^{m-1} \frac{(k-1) !}{(1 / 2)_{k}}\left(\frac{z}{z-1}\right)^{k}\right], \\
{ }_{2} F_{1}(1, b ; m ; z)=\frac{(z-1)^{m-1}}{(-b)_{m} z^{m-1}}\left[\Gamma(1-b)(1-z)^{-b}+z^{-1} \sum_{k=1}^{m-1}(-b)_{k}\left(\frac{z-1}{z}\right)^{-k}\right],
\end{gathered}
$$

one can derive elementary forms for the distribution of $|X / Y|$ when $m$ takes integer or half-integer values. This is illustrated in the corollaries below.

Corollary 3.2. If $m \geq 2$ is an integer, then (3.1) reduces to

$$
\begin{aligned}
F(z)=1-\frac{\left|1-c^{2}\right|^{m+1 / 2} \Gamma(2 m+1)}{\sqrt{\pi} 2^{2 m+1} \Gamma(m+1 / 2) \Gamma(m+3 / 2)}\{ & \frac{1}{\lambda b z-c} h\left(1-\frac{1}{(\lambda b z-c)^{2}}\right) \\
& \left.+\frac{1}{\lambda b z+c} h\left(1-\frac{1}{(\lambda b z+c)^{2}}\right)\right\},
\end{aligned}
$$

where

$$
h(z)=\frac{(1 / 2)_{m+1}(z-1)^{m}}{m ! z^{m+1}}\left\{2 \sqrt{z} \operatorname{arctanh}(\sqrt{z})+\sum_{k=1}^{m} \frac{(k-1) !}{(1 / 2)_{k}}\left(\frac{z}{z-1}\right)^{k}\right\}
$$

Corollary 3.3. If $m \geq 3 / 2$ is a half-integer, then (3.1) reduces to

$$
\begin{aligned}
F(z)=1-\frac{\left|1-c^{2}\right|^{m+1 / 2} \Gamma(2 m+1)}{\sqrt{\pi} 2^{2 m+1} \Gamma(m+1 / 2) \Gamma(m+3 / 2)}\{ & \frac{1}{\lambda b z-c} h\left(1-\frac{1}{(\lambda b z-c)^{2}}\right) \\
& \left.+\frac{1}{\lambda b z+c} h\left(1-\frac{1}{(\lambda b z+c)^{2}}\right)\right\},
\end{aligned}
$$



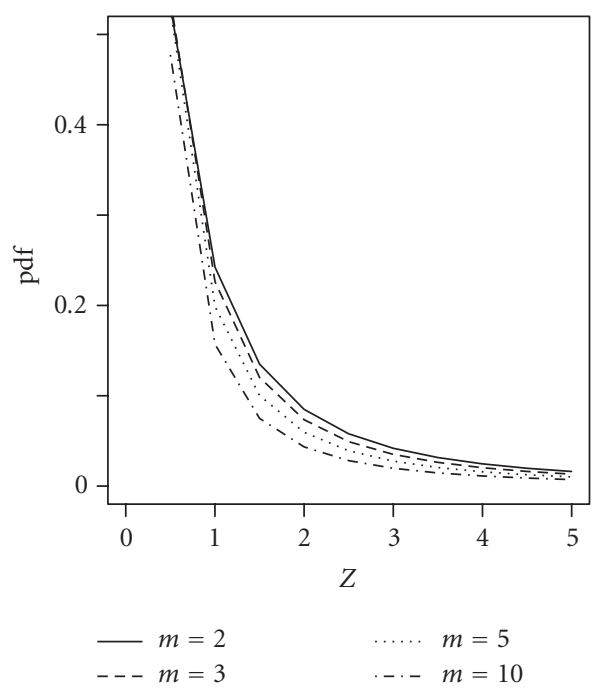

(a)

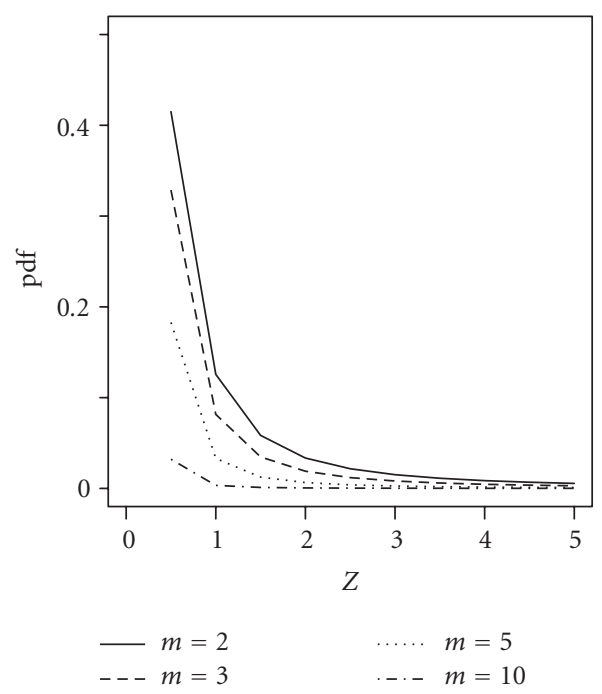

(c)

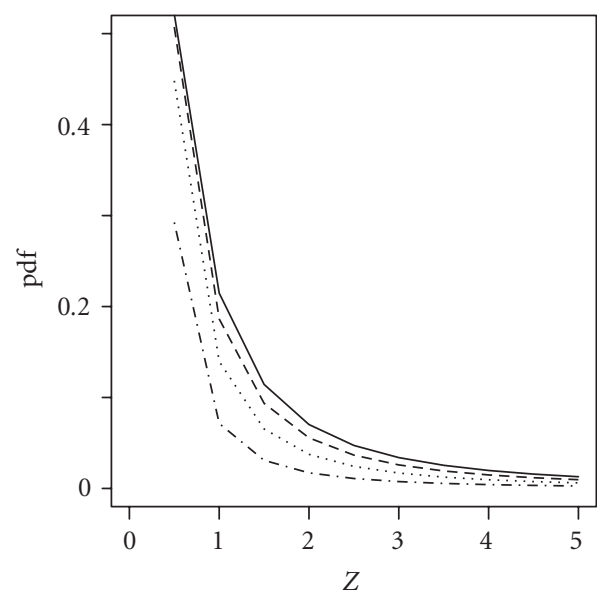

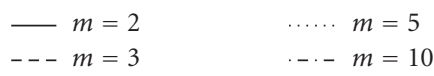

(b)

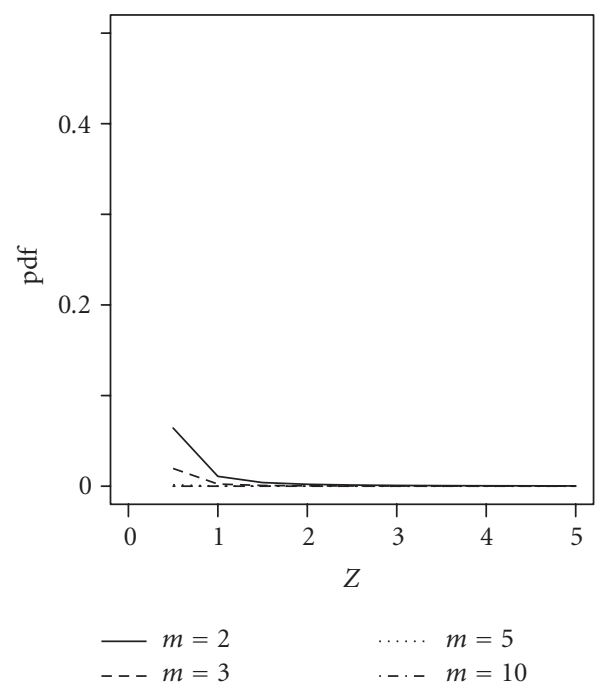

(d)

Figure 3.1. Plots of the pdf of (3.1) for $\lambda=1, b=1$, and (a) $c=0$; (b) $c=0.3$; (c) $c=0.6$; and (d) $c=0.9$. The four curves in each plot correspond to $m=2$ (solid curve), $m=3$ (curve of dots), $m=5$ (curve of dashes), and $m=10$ (curve of dots and dashes). 
where

$$
h(z)=\frac{(z-1)^{m+1 / 2}}{(-1 / 2)_{m+3 / 2} z^{m+1 / 2}}\left\{\sqrt{\pi}(1-z)^{-1 / 2}+\frac{1}{z} \sum_{k=1}^{m+1 / 2}(-1 / 2)_{k}\left(\frac{z-1}{z}\right)^{-k}\right\} .
$$

Figure 3.1 illustrates possible shapes of the pdf of $|X / Y|$ for a range of values of $c$ and $m$. The densities are unimodal and the effect of the two parameters on the shape of the densities is evident.

\section{Acknowledgment}

The author would like to thank the referee and the editor for carefully reading the paper and for their great help in improving the paper.

\section{References}

[1] M. S. Abu-Salih, Distributions of the product and the quotient of power-function random variables, Arab J. Math. 4 (1983), no. 1-2, 77-90.

[2] A. P. Basu and R. H. Lochner, On the distribution of the ratio of two random variables having generalized life distributions, Technometrics 13 (1971), 281-287.

[3] R. P. Bhargava and C. G. Khatri, The distribution of product of independent beta random variables with application to multivariate analysis, Ann. Inst. Statist. Math. 33 (1981), no. 2, 287-296.

[4] A. Erdélyi, W. Magnus, F. Oberhettinger, and F. G. Tricomi, Higher Transcendental Functions. Vol. I, Robert E. Krieger Publishing, Florida, 1981.

[5] M. S. Feldstein, The error of forecast in econometric models when the forecast-period exogenous variables are stochastic, Econometrica, Econometric Society 39 (1971), no. 1, 55-60.

[6] I. S. Gradshteyn and I. M. Ryzhik, Table of Integrals, Series, and Products, Academic Press, California, 2000.

[7] H. G. Grubel, Internationally diversified portfolios: welfare gains and capital flows, American Economic Review 58 (1968), no. 5, 1299-1314.

[8] H. L. Hartar, On the distribution of Wald's classification statistic, Ann. Math. Statistics 22 (1951), $58-67$.

[9] D. L. Hawkins and C.-P. Han, Bivariate distributions of some ratios of independent noncentral chi-square random variables, Comm. Statist. A-Theory Methods 15 (1986), no. 1, 261277.

[10] P. J. Korhonen and S. C. Narula, The probability distribution of the ratio of the absolute values of two normal variables, J. Statist. Comput. Simulation 33 (1989), no. 3, 173-182.

[11] S. Kotz, T. J. Kozubowski, and K. Podgórski, The Laplace Distribution and Generalizations, Birkhäuser Boston, Massachusetts, 2001.

[12] N. N. Lebedev, Special Functions and Their Applications, Dover Publications, New York, 1972.

[13] H. J. Malik and R. Trudel, Probability density function of the product and quotient of two correlated exponential random variables, Canad. Math. Bull. 29 (1986), no. 4, 413-418.

[14] G. Marsaglia, Ratios of normal variables and ratios of sums of uniform variables, J. Amer. Statist. Assoc. 60 (1965), 193-204.

[15] T. Pham-Gia, Distributions of the ratios of independent beta variables and applications, Comm. Statist. Theory Methods 29 (2000), no. 12, 2693-2715.

[16] H. Podolski, The distribution of a product of $n$ independent random variables with generalized gamma distribution, Demonstratio Math. 4 (1972), 119-123.

[17] S. J. Press, The t-ratio distribution, J. Amer. Statist. Assoc. 64 (1969), 242-252. 
402 On the product and ratio of Laplace and Bessel random variables

[18] S. B. Provost, On the distribution of the ratio of powers of sums of gamma random variables, Pakistan J. Statist. 5 (1989), no. 2, 157-174.

[19] A. P. Prudnikov, Yu. A. Brychkov, and O. I. Marichev, Integrals and Series. Vol. 1, Gordon \& Breach Science Publishers, New York, 1986.

[20] Integrals and Series. Vol. 2, Gordon \& Breach Science Publishers, New York, 1986.

[21]_, Integrals and Series. Vol. 3, Gordon \& Breach Science Publishers, New York, 1986.

[22] P. N. Rathie and H. G. Rohrer, The exact distribution of products of independent random variables, Metron 45 (1987), no. 3-4, 235-245.

[23] A. M. Rugman, International Diversification and the Multinational Enterprise, D. C. Heath, Massachusetts, 1979.

[24] H. Sakamoto, On the distributions of the product and the quotient of the independent and uniformly distributed random variables, Tôhoku Math. J. 49 (1943), 243-260.

[25] S. M. Shcolnick, On the ratio of independent stable random variables, Stability Problems for Stochastic Models (Uzhgorod, 1984), Lecture Notes in Math., vol. 1155, Springer, Berlin, 1985, pp. 349-354.

[26] M. D. Springer and W. E. Thompson, The distribution of products of beta, gamma and Gaussian random variables, SIAM J. Appl. Math. 18 (1970), 721-737.

[27] B. M. Steece, On the exact distribution for the product of two independent beta-distributed random variables, Metron 34 (1976), no. 1-2, 187-190 (1978).

[28] A. Stuart, Gamma-distributed products of independent random variables, Biometrika 49 (1962), $564-565$.

[29] J. Tang and A. K. Gupta, On the distribution of the product of independent beta random variables, Statist. Probab. Lett. 2 (1984), no. 3, 165-168.

[30] C. M. Wallgren, The distribution of the product of two correlated t variates, J. Amer. Statist. Assoc. 75 (1980), no. 372, 996-1000.

Saralees Nadarajah: Department of Statistics, University of Nebraska, Lincoln, NE 68583, USA

E-mail address: snadarajah2@unl.edu 


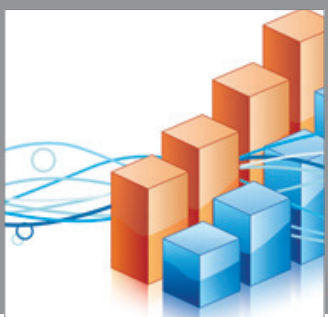

Advances in

Operations Research

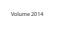

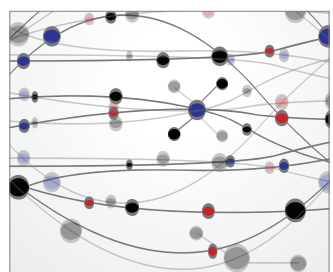

\section{The Scientific} World Journal
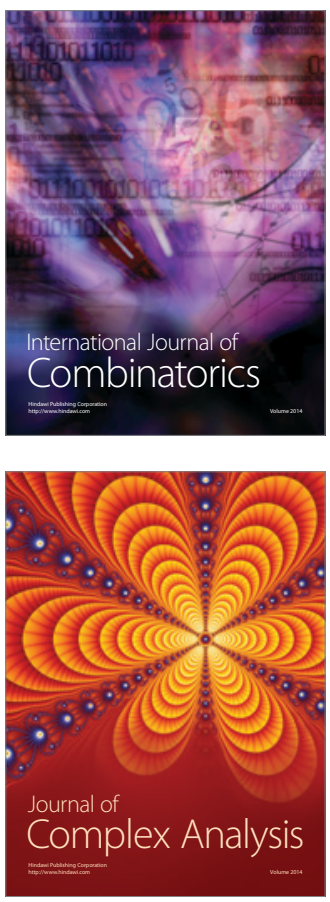

International Journal of

Mathematics and

Mathematical

Sciences
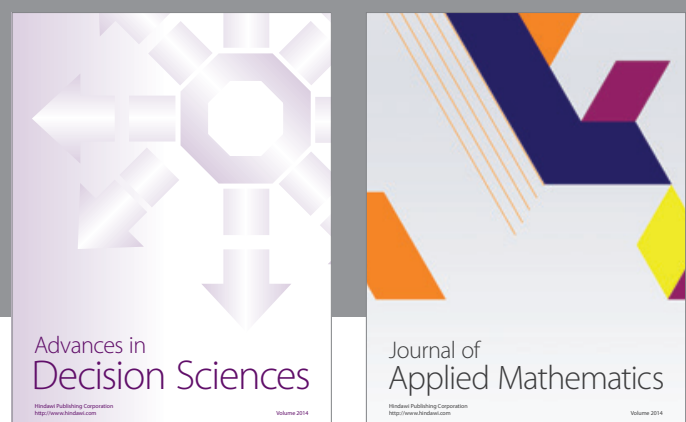

Journal of

Applied Mathematics
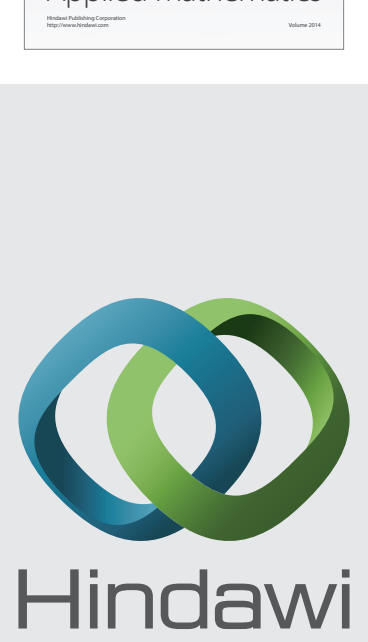

Submit your manuscripts at http://www.hindawi.com
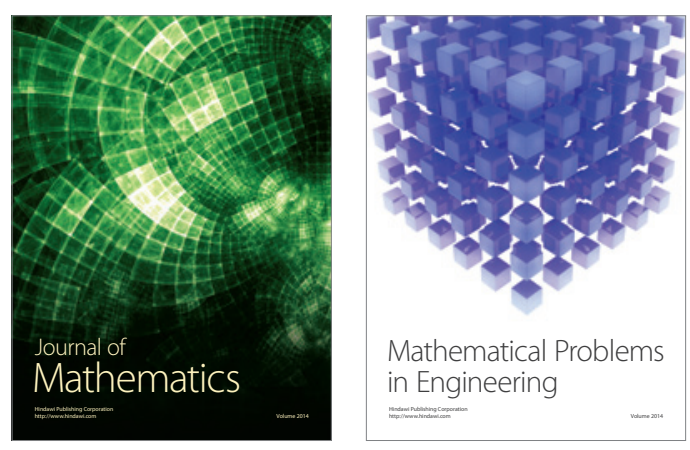

Mathematical Problems in Engineering
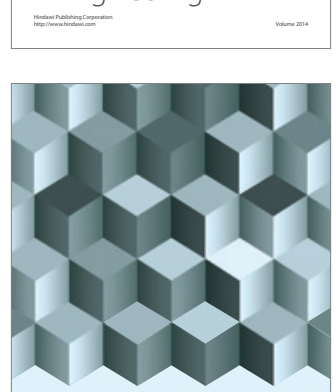

Journal of

Function Spaces
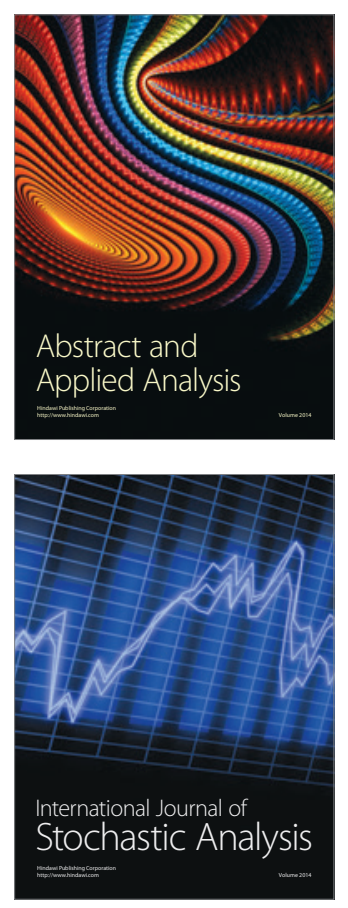

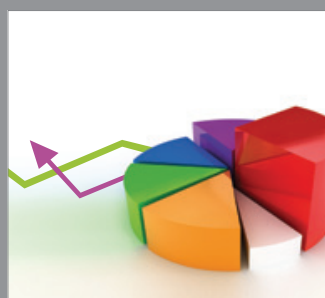

ournal of

Probability and Statistics

Promensencen
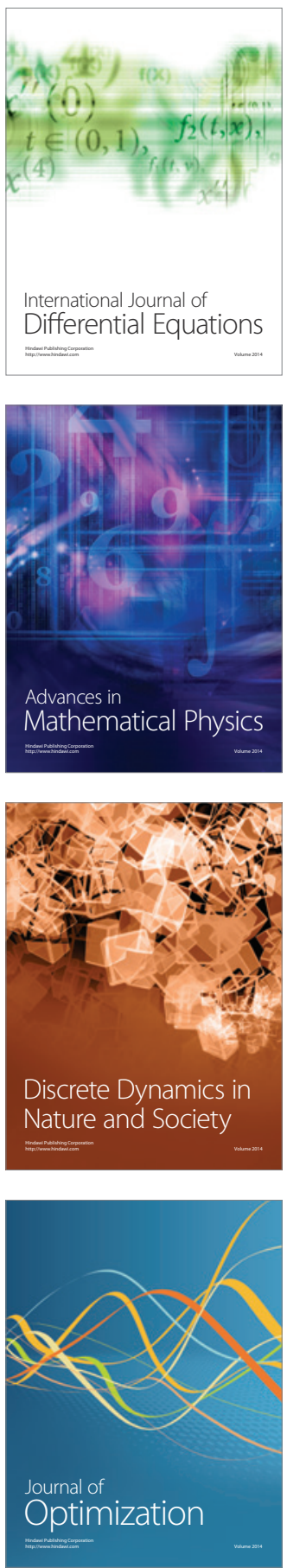